\title{
Engineering and Assembly of Protein Modules into Functional Molecular Systems
}

\author{
Stephan Hirschia, Mirko Stauffer ${ }^{\mathrm{a}}$, Daniel Hardera ${ }^{\mathrm{a}}$, Daniel J. Müller ${ }^{\mathrm{b}}$, Wolfgang Meier ${ }^{\mathrm{c}}$, \\ and Dimitrios Fotiadis ${ }^{\star a}$
}

\begin{abstract}
Synthetic biology approaches range from the introduction of unique features into organisms to the assembly of isolated biomacromolecules or synthetic building blocks into artificial biological systems with biomimetic or completely novel functionalities. Simple molecular systems can be based on containers on the nanoscale that are equipped with tailored functional modules for various applications in healthcare, industry or biological and medical research. The concept, or vision, of assembling native or engineered proteins and/ or synthetic components as functional modules into molecular systems is discussed. The main focus is laid on the engineering of energizing modules generating chemical energy, transport modules using this energy to translocate molecules between compartments of a molecular system, and catalytic modules (bio-)chemically processing the molecules. Further key aspects of this discourse are possible approaches for the assembly of simple nanofactories and their applications in biotechnology and medical health.
\end{abstract}

Keywords: Membrane protein · Molecular systems engineering · Nanofactory · Protein engineering Synthetic biology

\section{Introduction}

Synthetic biology is a highly interdisciplinary field at the interface of biology, chemistry and engineering. It aims at designing, creating and manipulating existing or synthetic biological systems for applications in healthcare, industry or biological and medical research. The two major approaches in synthetic biology are termed 'top-down' and 'bottom-up'.[1] 'Top-down' refers to the use of genetic engineering and the introduction of natural and artificial molecules into living organisms to create novel properties or functions. 'Bottom-up' on the other hand describes the process of building artificial biological systems from individual biological and/or synthetic components that mimic a natu-

\footnotetext{
${ }^{\star}$ Correspondence: Prof. Dr. D. Fotiadis ${ }^{a}$ E-mail: dimitrios.fotiadis@ibmm.unibe.ch anstitute of Biochemistry and Molecular Medicine University of Bern

Bühlstrasse 28, CH-3012 Bern

bDepartment of Biosystems Science and Engineering

ETH Zürich, $\mathrm{CH}-4058$ Basel

'Department of Chemistry

University of Basel, $\mathrm{CH}-4056$ Basel
}

ral function or exhibit functionalities not found in nature. Advances in recombinant DNA technologies have opened the way for rational metabolic engineering, i.e. for the 'top-down' manipulation of organisms. Enzymes from various origins can be reassembled into an organism to produce desired non-native secondary metabolites with high yields. This approach is most suitable for the production of chemically complex molecules, which are difficult to synthesize using conventional chemistry, and with low abundance in natural sources. One such example are terpenoids, which are used as precursor compounds to manufacture a variety of medicines including antimalarial and anticancer drugs as well as flavors and fragrances. An Escherichia coli strain was for example engineered to produce amorphadiene by introducing a mevalonate isoprenoid pathway through implementation of two synthetic operons with genes derived from yeast. ${ }^{[2]}$ Great progress has also been made in emulating properties and functions of living systems by assembling synthetic molecular systems from either synthetic organic molecules and/or functional biomacromolecules isolated from natural sources or overexpressing recombinant microorganisms. The synthetic approach can in many cases help to unravel biological working mechanisms by simplifying a complex phenomenon to its core components and investigating their interactions. 'Bottom-up' assembly can be useful to support conclusions drawn based on conventional analysis about multi- component biological processes, e.g. if a subgroup of components is able to achieve a certain assigned function. In this context, the molecular mechanisms of bacterial cell division have been investigated by reconstituting isolated components in biomimetic membranes (e.g. nanodiscs, supported bilayers and vesicles) and could thus be characterized. ${ }^{[3]} \mathrm{An}$ example is the reconstitution of membrane targeted FtsZ, a key protein component of the bacterial cell division machinery, into tubular vesicles. FtsZ alone assembled to form a ring inside the vesicles that resulted in observable constriction of the vesicles similar to the process of bacterial cell division. ${ }^{[4]}$

The 'bottom-up' assembly of isolated biomolecules and synthetic components is of major significance and interest as it can be used to create biomimetic systems with novel functionalities. Such molecular systems can be built 'bottom-up' as membrane surfaces or vesicular structures that constitute containers in the nanoscale, hereinafter referred to as nanocontainers, equipped with specialized functional modules (Fig. 1a and b). Modules employed are of biological and/or chemical nature, and can be engineered to meet the requirements of the molecular system. Functional membranes on nanofabricated surface structures create two-dimensionally organized nanocontainers (Fig. 1a) as alternatives to classical vesicular containers (Fig. 1b). Natural and synthetic lipids are readily available to build functionalized membrane surfaces and lipid vesicles and can also be replaced 
by lipid-mimicking block copolymers that are chemically more stable (Fig. 1b). ${ }^{[5]}$ However, incorporation of functional modules into such polymer surfaces or polymerosomes is not straightforward and therefore liposomes are frequently preferred for initial studies. Since proteins are among the biomolecules with the most diverse functions, they are excellent module candidates, which can further be engineered using state-of-the-art molecular biology techniques. To make 'bottomup' assembled molecular systems functional, energizing modules are essential that provide chemical energy for driving energy-dependent modules. Living organisms use electrochemical gradients across membranes and hydrolysis of high-energy phosphate compounds to make endergonic reactions possible. Energy is supplied by membrane-bound light-driven ion pumps, transmembrane ATPases or both combined. ${ }^{6]}$ Membrane transport proteins, briefly transporters, can be used to mediate the translocation of reactants and products between compartments of molecular systems, e.g. between the inside and outside of vesicles or other containers. According to the abundance of reactants and products, transporters with high affinity but low transport capacity or low affinity and high transport capacity for specific substrate molecules have to be used. Finally, soluble modules (enzymes or chemical catalysts) encapsulated inside the containers allow the processing of incoming or stored reactants. Enzymes but also chemical catalysts can be enantioselective and both usually have high turnover rates. The selectivity of enzymes for their substrates is in many cases vastly superior to that of common chemical catalysts. However, for numerous reactions nature offers no suitable enzymatic reaction and synthetic catalysts become indispensable. Possible types of reactions that can be performed inside nanocontainers are degradation, modification or ligation of substrates. An artificial enantioselective enzyme is created by accommodating a biotinylated synthetic catalyst in the biotin binding pocket of either avidin or streptavidin where it is provided with a chiral environment. ${ }^{\text {[7] }}$ Complementing a synthetic catalyst with the framework of a protein can be an elegant way to combine a synthetic catalyst's unique reaction mechanism with the chiral environment of a protein. Catalysis can be severely diminished by unspecific interactions with non-substrate molecules and the low concentration of the substrate in the environment. Compartmentalization of the reaction into a small volume with only selective permeability, i.e. with specific transport modules, is thus essential, solving the problems of unspecific interactions and low concentration simultaneously.

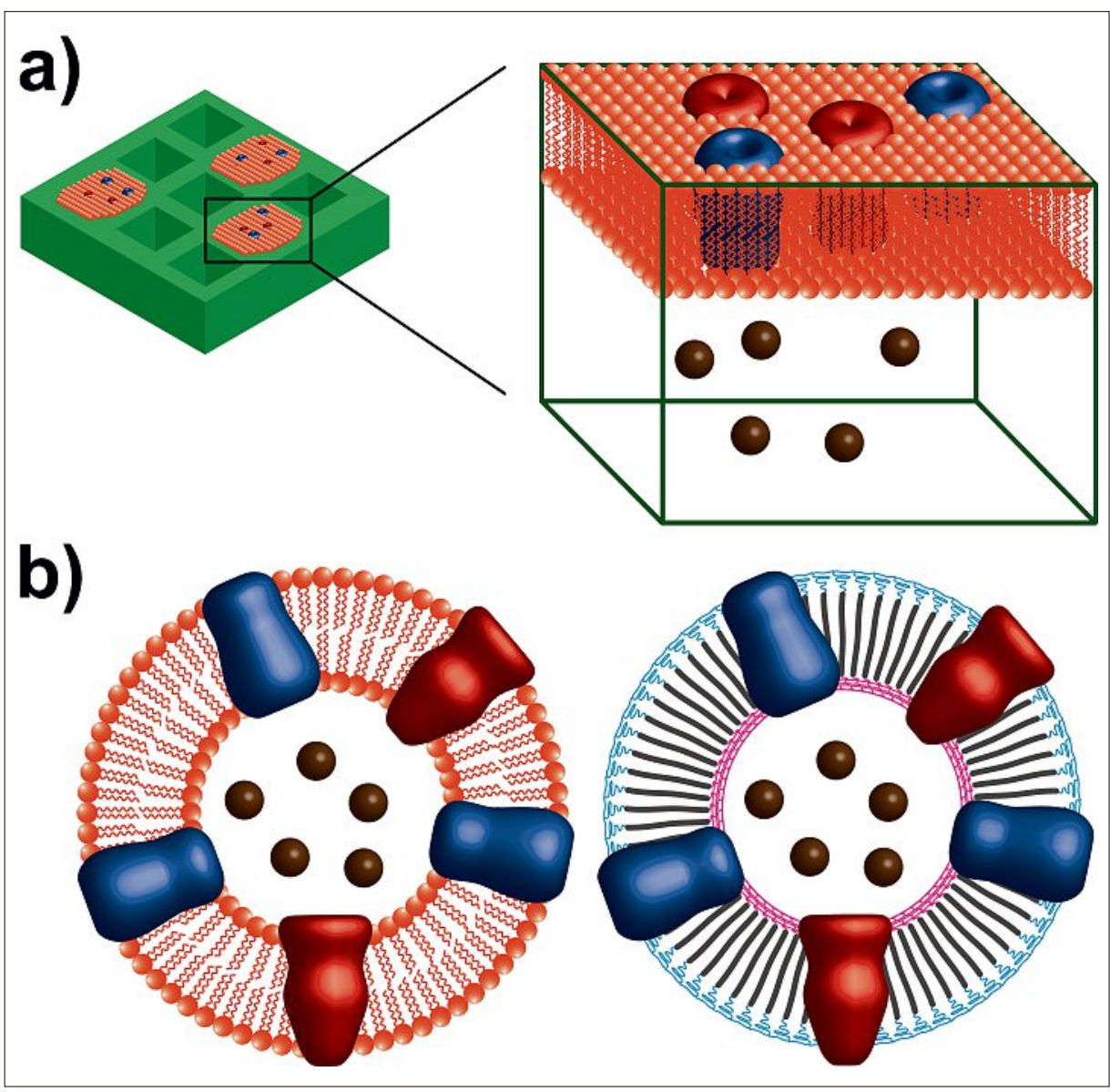

Fig. 1. Different types of functional molecular systems based on either functionalized surfaces (a) or vesicular structures (b). Micro- and nanofabricated surfaces (green) are patterned to create compartments with defined properties when enclosed by a functional surface such as a membrane protein containing lipid bilayer. Membranes can be made of natural or synthetic lipids (b, left panel) or block copolymers (b, right panel). Incorporated functional modules include energy providing modules (red), energy-dependent transport modules (blue) and soluble catalytic modules (brown)

\section{Engineering of Protein Modules}

The primary components of the heredescribed simple functional molecular system are energizing and transport modules. Application of light-driven ion pumps as energizing modules is more straightforward than that of using complex multisubunit ATPases, since energy in form of light is easily supplied to a system and can be converted by a single membrane protein to generate an ion gradient. This reduces the complexity level of reconstituting the energy converting module and of the provided energy forms to be used by other modules. Detailed knowledge concerning the function of protein modules is a prerequisite for their efficient use. Furthermore, the availability of their structures is advantageous for structure-based mutagenesis and functional engineering. Henceforth, we will focus on well-studied light-driven proton pumps (LDP) such as bacteriorhodopsin $(\mathrm{BR})^{[8]}$ and proteorhodopsin $(\mathrm{PR})^{[9]}$ that establish proton gradients across membranes. This proton gradient can then be used to power proton-driven transporters.
The proton translocation mechanism of LDPs is initiated by the absorption of photons of a specific wavelength by a covalently bound all-trans retinal. It has been shown that this specific absorption wavelength can be manipulated by mutating the LDPs. ${ }^{[10]}$ Amino acid residues in the vicinity of the retinal cofactor directly affect the electronic structure of the retinal and thus its absorptive properties. This raises the possibility for a molecular system containing two different versions of spectrally tuned ion pumps to have two distinct functions or to regulate its function depending on the used wavelength.

Transporter modules need to guarantee specific translocation of the desired solute across the container's membrane. A plethora of membrane transport proteins with different substrate classes, e.g. amino acids, peptides, sugars, nucleotides and fatty acids, occur in nature. However, only a relatively small number of transporters has been identified and characterized in sufficient detail to facilitate rational protein engineering and implementation into bottom-up assembled molecular systems. 
In cases where information on the atomic structure is available, the specificity and/ or affinity of a given transporter for its substrates can be tuned by structure-based mutagenesis. ${ }^{[11]}$ Crucial amino acid side chains in the binding pocket of the transporter that interact with the substrate are mutated to alter the binding properties. For example, important electrostatic interactions between the substrate and the amino acid side chains can be affected by substituting a charged with an uncharged residue. Another option is the site-specific introduction of a bulky or a small side chain to render the binding pocket inaccessible or accessible for larger substrates, respectively.

An intriguing possibility is the implementation of an 'on-off' switch that allows full control over the activity of the functional modules. Chemically switchable versions of proteins can be based on cysteine mutants that are reversibly modified by bulky or charged sulfhydryl reactive reagents. ${ }^{[12,13]}$ The chemical modification interferes with the mechanism of action, thus inhibiting protein function. Recently, we have engineered a chemical 'on-off' switch into the LDP proteorhodopsin, thus extending its versatility and applications in molecular factories. ${ }^{[14]}$

\section{Assembly of Functional Molecular Systems}

Assembled molecular systems are based on structures such as surfaces that enclose a compartment (Fig. 1a) or liposomes/polymerosomes (Fig. 1b). Functional modules, in this case membrane proteins and soluble catalysts (of biological or chemical nature), need to be embedded in the membrane and inside the compartment, respectively. Lipids, block copolymers and membrane proteins are all amphiphilic molecules that require detergents to stay soluble in aqueous environments. Classical approaches to reconstitute membrane proteins into liposomes include different methods of controlled detergent removal, such as dilution, dialysis, Biobeads, gel filtration and cyclodextrins. [15-17] Removal of the stabilizing detergents promotes the interaction of membrane proteins with the respective lipids or block copolymers, eventually yielding protein reconstituted into liposomes or polymerosomes. Reconstitution can occur either from protein-lipid-detergent ternary complexes with detergent solubilized lipids or from solubilized protein and preformed liposomes that are destabilized upon addition of detergent. Both methods yield functional proteoliposomes, but the orientation of the incorporated membrane proteins can vary depending on the employed method of reconstitution.[17] Membrane protein orientation in preparations from fully solubilized material is basically random, but in some cases can have preferences for either side. ${ }^{[18,19]}$ A more pronounced asymmetric distribution is observed with preformed liposomes. This is due to perferential interactions of detergent-solubilized membrane proteins with the liposome surface. For example, a membrane protein with a bulky and a small hydrophilic domain on the cytoplasmic and extracellular side, will preferentially insert into liposomes exposing the bulky portion to the outside leading to directed incorporation of the protein into the lipid membrane. A functional short-circuit arises from the symmetrical distribution of energizing modules in nanocontainer membranes (Fig. 2). One possibility to resolve such short-circuits is the application of protein modules endowed with a chemical 'on-off' switch based on the modification of cysteine mutants by sulfhydryl reactive agents (see above Engineering of Protein Modules). Modules with undesired orientation could be simply switched off, thus introducing an asymmetry into the membrane. Only energy providing modules require orientation as the introduced asymmetry is imposed on subsequent modules by the established electrochemical gradient.

'Nanofactories' of higher complexities can be formed by fusing individual proteoliposomes containing single or several protein modules, or produced through coreconstitution of several modules (simultaneous or sequential). The number of incorporated and connected functional modules is in principle only limited by their compatibility to each other's functional and physicochemical properties (e.g. preferences for particular lipids and block copolymers). It is thus imaginable that different combinations of functional molecular systems can eventually lead the way to first 'nanofacto- ries' or even 'nanocells' emulating partial cellular functions.

\section{Vision and Conclusion}

Based on the concepts of functional molecular systems as discussed above, a few examples are presented hereafter. For all applications involving enzymes, it is highly beneficial to work in confined and only selectively permeable compartments to enhance substrate turnover rates and reduce interference by unspecific compounds. Therefore, all of the following examples are based on nanocontainers accommodating biological and/or chemical modules.

An assembly of LDPs, proton-driven transporters and metabolizing enzymes in nanocontainers such as proteoliposomes or polymerosomes (Fig. 1b) could assume the role of a 'molecular hoover'. Such a system would be capable of generating an electrochemical gradient as energy source for the subsequent substrate translocation into the nanocontainer by proton-dependent transporters with high affinity for specific chemical compounds. Substrates could, for example, be specific pollutants that need to be removed from water supplies. Furthermore, a metabolizing module (e.g. a degrading enzyme or chemical catalyst) could be encapsulated within the 'molecular hoover' to further dispose of the solute after accumulation inside the container. Depending on the method used for the assembly of the system and on the resulting sidedness of the incorporated membrane protein modules, 'on-off' switchable LDPs might be required for the introduction of a functional asymmetry in the membrane and the prevention of a short-circuit (Fig. 2). Second, transporters with appropriate selectivity and high substrate affinity are necessary to efficiently

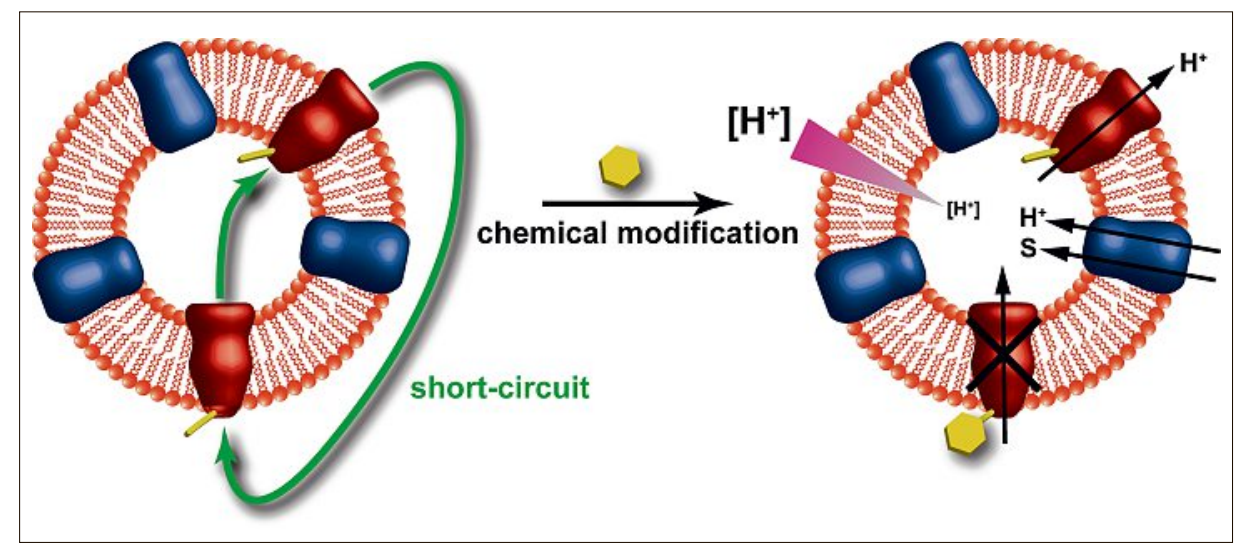

Fig. 2. Short-circuit created by the symmetrical distribution of functional modules inside a liposome-based molecular system. After chemical modification of the undesired population of energizing modules (red; here LDPs) using an implemented 'on-off' switch (yellow), an electrochemical gradient is established. Proton-driven symporters (blue) harness that power to selectively import a specific substrate (S) into the nanocontainer. 
catch and transport substrate present at low concentration in the bulk solution into the container. Finally, an enzyme or chemical catalyst inside the container is needed to degrade the substrate, thus promoting the uptake of further molecules.

The opposite, i.e. a molecular system with applications in the controlled release of compounds is conceivable. A molecule that has been loaded into a nanocontainer or is synthesized in situ could be released on command by simply illuminating the system. Similar to the previous example, such a molecular system comprises the same kind of modules, but in opposite orientation to enable the efflux of a substrate or product. Reactants that are co-encapsulated with a biological or chemical catalyst are converted to a desired product that can then be exported either continuously or on demand (e.g. by stimulation with light of a specific wavelength). This idea can of course be evolved into a more complex system, where any number of functional nanocontainers is connected in series like an assembly line. To this end, the compartments following the first require either two transporters, one to import the previous product as reactant and another to export the second product, or a specific exchanger (antiporter), that exchanges a reactant from the outside with a product from the inside. The system could also be designed in a way, where the second product is hydrophobic enough to simply diffuse through the vesicle membrane, without the need of product specific exporters. Such an assembly line enables the unattended execution of a multistep synthesis in one reaction chamber, without the need to separate the product from remaining reactions before continuing with the next reaction step.

As mentioned at the beginning, functional molecular systems as described here do not need to be solely based on vesicular structures but can also be built as functionalized surfaces. This results from the combination of micro- or nanofabricated surfaces with functional modules reconstituted into lipid or polymer membranes (Fig. 1a). Surfaces can be patterned in the nanoscale by existing lithographic methods providing a basis for the construction of highly sophisticated molecular assemblies when combined with the variety and complexity of biological and chemical modules at our disposal.

The road from the production and engineering of individual modules, through the assembly process towards functional molecular system as described here might seem long and tedious. We are, however, not only compensated for our efforts with the final products, i.e. the functional molecular system itself, but also with the advancement of fundamental knowledge and all innovations made along the way that can prove extremely valuable assets for synthetic biology. Some of the newfound methods can be refined and gradually developed into everyday laboratory techniques, as it was the case with genetic manipulation of microorganisms at the very beginning of synthetic biology, or bioengineering as it was called at that time. The diversity of molecular systems and their possible applications are virtually limitless ranging from drug delivery, controlling cellular systems, diagnostic devices, bioremediation, to the synthesis of chemical compounds and many more. Therefore, it is worthwhile investing in the advancement of synthetic biology, which has the great potential for improving human health and quality of life, advancing biological and medical research and giving industry new perspectives.

\section{Acknowledgments}

Financial support from the Universities of Bern and Basel, the ETH Zürich and the National Centre of Competence in Research Molecular Systems Engineering is gratefully acknowledged.
[1] S. A. Benner, A. M. Sismour, Nat. Rev. Genet. $\mathbf{2 0 0 5}, 6,533$

[2] V. J. J. Martin, D. J. Pitera, S. T. Withers, J. D. Newman, J. D. Keasling, Nat. Biotechnol. 2003 $21,796$.

[3] A. Martos, M. Jiménez, G. Rivas, P. Schwille, Trends Cell Biol. 2012, 22, 634.

[4] M. Osawa, D. E. Anderson, H. P. Erickson, Science 2008, 320, 792.

[5] M. Kumar, J. E. O. Habel, Y. Shen, W. P. Meier T. Walz, J. Am. Chem. Soc. 2012, 134, 18631.

[6] H. J. Freisleben, K. Zwicker, P. Jezek, G. John, A. Bettin-Bogutzki, K. Ring, T. Nawroth, Chem. Phys. Lipids 1995, 78, 137.

[7] C. Letondor, N. Humbert, T. R. Ward, Proc Natl. Acad. Sci. 2005, 102, 4683.

[8] J. K. Lanyi, Annu. Rev. Physiol. 2004, 66, 665.

[9] C. Bamann, E. Bamberg, J. Wachtveitl, C. Glaubitz, Biochim. Biophys. Acta - Bioenerg. 2014, 1837, 614

[10] S. Y. Kim, S. A. Waschuk, L. S. Brown, K.-H. Jung, Biochim. Biophys. Acta - Bioenerg. 2008, 1777,504

[11] R. Boggavarapu, J.-M. Jeckelmann, D. Harder, Z. Ucurum, D. Fotiadis, BMC Biol. 2015, 13, 58.

[12] M.-F. Tsai, Y. Fang, C. Miller, Biochem. 2012 $51,1577$.

[13] A. Karlin, M. H. Akabas, Methods Enzymol. $\mathbf{1 9 9 8}, 293,123$.

[14] D. Harder, S. Hirschi, Z. Ucurum, R. Goers, W. Meier, D. J. Müller, D. Fotiadis, Angew. Chem. Int. Ed. 2016, in press.

[15] L. Wang, L. Tonggu, Sci. China Life Sci. 2015, 58,66 .

[16] G. A. Signorell, T. C. Kaufmann, W. Kukulski, A. Engel, H.-W. Rémigy, J. Struct. Biol. 2007, 157, 321.

[17] J. L. Rigaud, M. T. Paternostre, A. Bluzat, Biochem. 1988, 27, 2677.

[18] H. S. Young, J. L. Rigaud, J. J. Lacapère, L. G. Reddy, D. L. Stokes, Biophys. J. 1997, 72, 2545.

[19] L. Wang, F. J. Sigworth, Nature 2009, 461, 292 